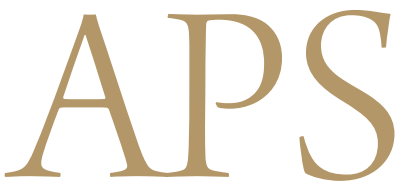

Archives of Plastic Surgery

\title{
Muscle-Sparing Blepharoplasty: A Prospective Left-Right Comparative Study
}

\author{
Lee Kiang ${ }^{1}$, Peter Deptula ${ }^{2}$, Momal Mazhar ${ }^{2}$, Daniel Murariu ${ }^{3}$, Fereydoun Don Parsa ${ }^{2}$ \\ ${ }^{1}$ Department of Ophthalmology, W.K. Kellogg Eye Center and Visual Sciences, University of Michigan, Ann Arbor, MI; ${ }^{2}$ Department of \\ Surgery, Division of Plastic Surgery, University of Hawaii, John A Burns School of Medicine, Honolulu, HI; ${ }^{3}$ Department of Plastic Surgery, \\ University of Virginia, Charlottesville, VA, USA
}

Background Standard upper blepharoplasty involves removal of both the skin and a portion of the underlying orbicularis oculi muscle. The senior author had observed sluggishness of eyelid closure, lagophthalmos as well as varying degrees of eye irritation in certain patients during the early postoperative period. He postulated that these findings could be due to orbicularis muscle excision. He therefore undertook a prospective study 27 years ago comparing standard blepharoplasty on one eyelid to skin-only excision on the fellow eyelid.

Methods A randomized, prospective, single-blinded study was designed using the fellow eye as an internal control. 22 patients undergoing upper blepharoplasty procedure requiring greater than $5 \mathrm{~mm}$ of skin resection and with no history of ophthalmologic disease, dry eye, or previous eyelid surgery were selected. Upper blepharoplasty was performed with skin-only removal on one side, and combined skin-muscle removal on the other side. Patients were evaluated until six months after surgery except for two patients who were lost to follow-up after three months. Sluggish eyelid closure, lagophthalmos, dry eye and aesthetic result were outcome measures scored by patient survey, the operating surgeon, and a blinded expert panel. Results There were comparable aesthetic outcomes in both eyelids. The incidence of sluggish eyelid closure, lagophthalmos and dry eye syndrome were significantly higher in eyelids where wide segments of muscle had been resected.

Conclusions Muscle-sparing upper blepharoplasty produces similar aesthetic outcomes as conventional blepharoplasty, while significantly reducing the complications of sluggish eyelid closure, lagophthalmos and dry eye disease. The authors therefore recommend muscle-sparing upper blepharoplasty.

Keywords Blepharoplasty / Ptosis / Dry eye syndromes

\author{
Correspondence: \\ Fereydoun Don Parsa \\ Department of Surgery, Division of \\ Plastic Surgery, University of Hawaii, \\ John A Burns School of Medicine, \\ 1329 Lusitana Street, Suite 807, \\ Honolulu, HI 96813, USA \\ Tel: $+1808526-0303$ \\ Fax: $+1808536-8836$ \\ E-mail: fdparsa@gmail.com
}

No potential conflict of interest relevant to this article was reported.

Received: 18 Jun 2014 • Revised: 22 Jul 2014 • Accepted: 26 Jul 2014

pISSN: 2234-6163 • elSSN: 2234-6171 • http://dx.doi.org/10.5999/aps.2014.41.5.576• Arch Plast Surg 2014;41:576-583

\section{INTRODUCTION}

Conventional upper blepharoplasty typically involves removal of skin, orbicularis oculi muscle and, frequently, portions of the periorbital fat [1-3]. The levator, which opens the eyelid, and the orbicularis oculi, which closes the eyelid, function in concert to protect the conjunctiva and cornea [4]. Any injury to these structures creates an imbalance that may vary from lagophthalmos to ptosis of the upper eyelid with troublesome clinical manifestations [5]. The senior author noted that following stan- 
dard upper blepharoplasty, sluggishness in lid closure was often associated with lagophthalmos and occasional dry eye symptoms when relatively large segments of the underlying muscle had been excised. Based on such observations, this prospective study was designed in 1985 and continued to 2013. Both aesthetic and functional outcomes were evaluated when only skin was removed on one side and skin with muscle was removed on the contralateral eyelid.

The literature is generally silent on the effect that removal or preservation of the orbicularis muscle has on the aesthetic or functional outcome [6-9]. Fagien [2] in 2002, and Rohrich et al. [1] in 2004, advocated muscle sparing for physiologic and aesthetic reasons. Rohrich et al. [1] recommends the excision of "redundant orbicularis" once skin has been removed and preservation of the muscle in "non-redundant cases". It has been argued by few authors that orbicularis removal may improve aesthetic outcomes $[10,11]$. A recent prospective left-right study found that sparing of the orbicularis oculi decreased postoperative pain, edema, itching and hematoma, while producing good aesthetic results when compared to conventional surgery in the fellow eye [12].

Postoperative complications of upper blepharoplasty include swelling, pain, peribulbar hematoma, dry eye, lagophthalmos, chemosis, ptosis, granulomas, and webbing [1-5]; rare complications include ectropion, vision loss, retrobulbar hematoma and blindness [5]. Lagophthalmos that follows aesthetic upper blepharoplasty is frequently due to excessive removal of skin as shown in Fig. 1. The photograph was taken before this study was undertaken and depicts a patient attempting to gently close her eyelids at six months following bilateral "standard" skinmuscle excision. Despite a normal Bell's phenomenon, she remained symptomatic with eye irritation and redness, requiring eye lubricants and moisturizers during five years of follow-up. During this period, she showed no noticeable improvement of sluggish lid closure, but lagophthalmos improved significantly and was no longer present after a year of follow-up. At that time, it was the senior author's (F.D.P.) opinion that excessive skin removal was the primary cause of the patient's condition. Even so, he questioned whether the patient would have developed postoperative lagophthalmos, sluggish lid closure and dry eye symptoms if the orbicularis muscle had not been removed during surgery. This question became the primary motivation to undertake the present study in 1985 . This prospective study suggests that relatively wide but incomplete orbicularis muscle excision may in fact contribute to sluggish lid closure, lagophthalmos and dry eye disease.

Our goal in undertaking this study was to investigate whether upper blepharoplasty with sparing of orbicularis oculi could de- crease the incidence of postoperative sluggish eyelid closure, lagophthalmos and dry eye, while producing equivalent aesthetic outcomes to surgery with muscle resection.

\section{METHODS}

\section{Patient selection}

From 1985 to 2013, 22 patients requiring greater than $5 \mathrm{~mm}$ of skin resection underwent bilateral upper blepharoplasties with removal of muscle on one side and leaving muscle intact on the other side. Although the senior author has a busy aesthetic blepharoplasty practice, only 22 patients, less than $0.5 \%$ of the total number of eyelid procedures performed by him, knowingly consented to undergo these separate procedures, as was clearly documented on each consent form; these patients are reviewed in this report. Patients requiring simultaneous brow lift or with a history of previous eyelid surgery, ophthalmic surgery, dry eye disease or use of eye drops for symptoms such as foreign body sensation, tearing, itchiness or redness were excluded from the study. Botulinum toxin injection six months before surgery and during the study period was also exclusion criteria.

\section{Surgical techniques}

All procedures were performed by the same surgeon using the same operative technique in all patients. Upper eyelids were marked in a standard fashion corresponding to the amount of skin to be excised as shown in Fig. 2. Skin and the underlying portion of orbicularis oculi were excised en bloc from one eyelid of each patient in standard upper blepharoplasty fashion, while skin alone was excised from the lid of the fellow eye in alternating the sides in consequent patients (Figs. 2, 3). It was not possible to delineate, measure or document the precise outlines of the inner canthal or extra canthal orbicularis during the procedures. It is highly likely that in patients undergoing wider skinmuscle excision, it was also necessary to remove varying portions of inner and extra canthal orbicularis. This removal would certainly affect patients' eyelid closure mechanics.

Seven patients underwent concurrent lower blepharoplasties using fat-preserving capsulopalpebral repair with total preservation of the lower eyelid orbicularis muscle and lateral musculoplasty. This technique is identical to that described in our published case series in 2008 [13]. In this previous study, as well as in the present paper, no findings suggestive of "fish-mouthing", "rounding", scleral show or ectropion were noted [13].

\section{Outcomes}

Patients were evaluated both by the operating surgeon, who objectively assessed for any postoperative complications, and by a 


\section{Fig. 1. Standard upper blepharoplasty}

This 60-year-old patient had undergone "standard" upper blepharoplasties six months previously with removal of skin and muscle. She developed sluggish lid closure, lagophthalmos and eye irritation after surgery.

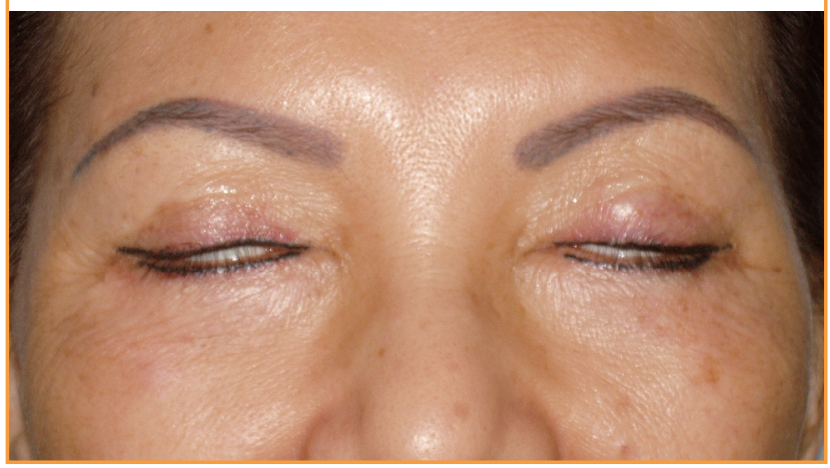

Fig. 2. Preoperative marking

Patient has been marked for skin-only excision of upper eyelid skin on one side and combined skin-muscle excision of the contralateral eyelid.

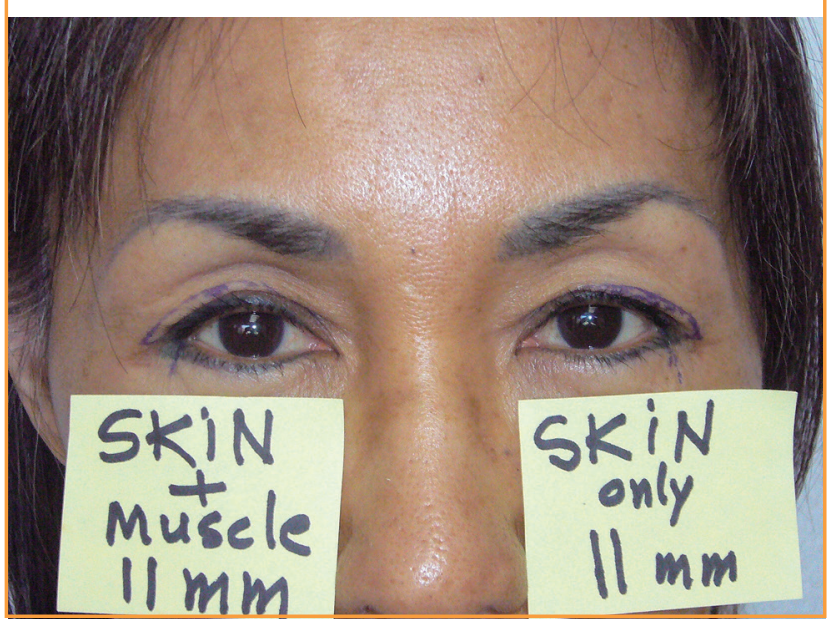

panel of actively practicing plastic surgeons, who evaluated for aesthetic outcome. At one week, two weeks, six weeks, three months and six months postoperatively, the operating surgeon examined each patient for the presence or absence of complications. Lagophthalmos was measured in millimeters in extreme downward gaze (Fig. 4), while sluggish lid closure was evaluated by asking patients to close their eyes as though sleeping or by having patients blink normally. Photographs were taken before surgery (Fig. 2), at the completion of the procedure (Fig. 3), as well as at three months and at six months following the operation (Figs. 5-10). Any degree of sluggish lid closure, lagophthalmos as well as symptoms and findings of dry eye disease were documented as either present or absent (Table 1). If lagopthalmos was identified, the patient was also examined for a normal Bell's phenomenon. Patients with persistent symptoms related to dry eye were followed for a longer time period.

\section{Fig. 3. Intraoperative left-right comparison}

Intraoperative photograph of a patient at the completion of upper blepharoplasties after skin-only excision of the right upper eyelid and combined skin-muscle excision of the left eyelid in a 54-yearold patient. Tarsal fixation was also performed.

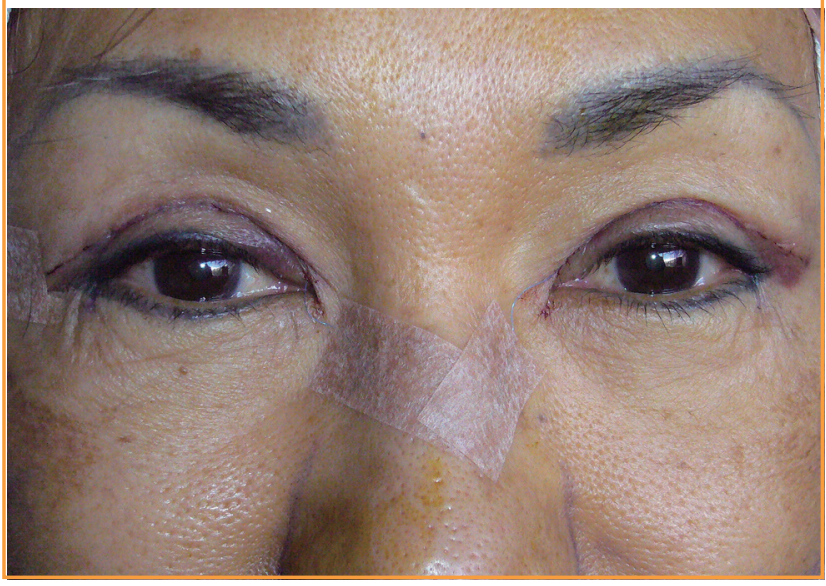

Fig. 4. Postoperative dynamic evaluation

Postoperative photograph of a 72-year-old patient taken at extreme downward gaze at one week after surgery. Lagophthalmos is present on the right eyelid where $13 \mathrm{~mm}$ of combined skin-muscle was removed. Lower blepharoplasties were also performed. Note the patient's unsuccessful attempt to close her eyes forcefully.

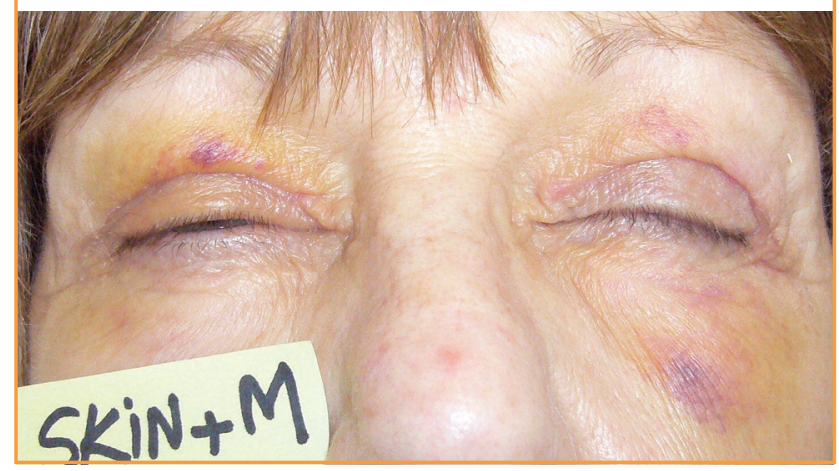

An anonymous patient satisfaction survey was conducted using questionnaires. These surveys were provided to all patients at six months after surgery. Patients were queried regarding age, date of surgery, ethnicity, satisfaction with outcome, any perceived difference between eyelids, preference of appearance of one side over the other, foreign body sensation, tearing, burning and eye irritation.

Evaluations were also made by three actively practicing plastic surgeons who examined patients' before and after photographs at six months after surgery. If the patient was lost to follow-up, photographs from the latest office visit were used. The evaluators were blinded to the type of repair, but were informed that none of the patients had undergone concurrent brow lift-an exclusion criteria-or had previous eyelid or brow lift operations. They were asked whether the eyelids had an outcome they would consider 


\section{Fig. 5. Preoperative photograph}

Before photograph of a 58 -year-old patient undergoing bilateral upper and lower blepharoplasties. Skin-only resection was randomly assigned to one upper eyelid and muscle-skin resection assigned to the other lid.

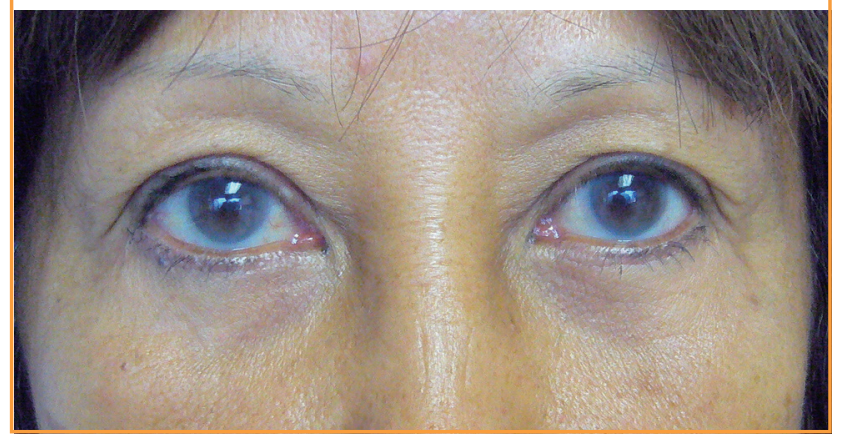

Fig. 6. Postoperative left-right comparison

Postoperative photograph of the patient in Fig. 5 six months after 8 $\mathrm{mm}$ skin-only excision of the left lid and combined skin-muscle excision of the right upper eyelid. Tarsal fixation was done on both sides. Concurrent lower blepharoplasties were also performed. This patient had no postoperative complications.

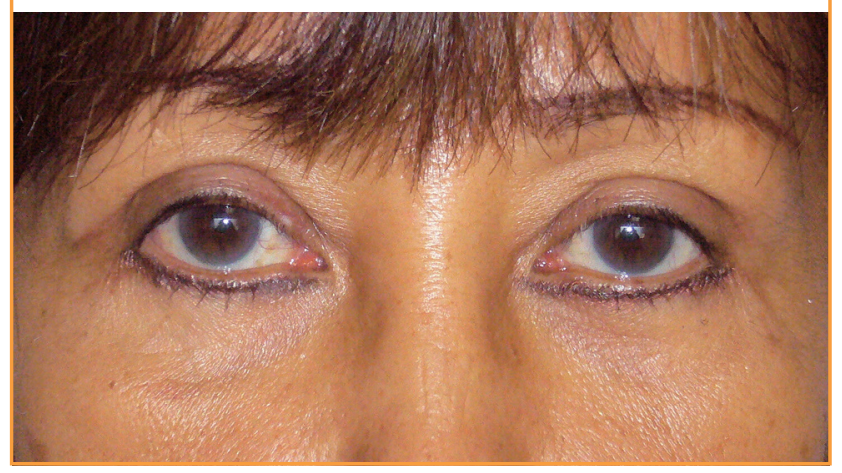

in their own practice as "good and comparable between right and left sides"; "good, but not comparable between right and left side"; and "poor on one or on both sides" and to grade each eyelid separately (Table 2). Evaluation by the same expert panel of surgeons took place after all photographs for the 22 subjects were obtained to ensure consistency of evaluation.

Statistical analyses were conducted using Fisher's exact test with mid-p, two-sided values of $\mathrm{P}<0.05$ considered significant.

\section{RESULTS}

The duration of the study was 27 years from 1985 to 2013 . The study population of one male and 21 female subjects had an age mean of 62, a median of 63.5, and a range of 44 to 78 years. In regards to ethnicity, 11 patients self-identified as Asian, four identified as mixed, and seven as Caucasian. Two female patients (9\%) were lost to follow-up after their three months postoperative visit. The remaining 20 patients or $91 \%$ were followed up to six months after surgery. Tarsal fixation to improve the supratarsal crease definition or to create one when the crease was absent, was performed concurrent to standard blepharoplasty in 11 (50\%) patients; two of these patients self-identified as mixedAsian and other ethnicity, and nine identified as Asian. The mean time of office follow-up was 5.8 months.

During the office follow-up visits at one week after surgery, sluggish eyelid closure was noted in seven patients (32\%). Sluggishness of lid closure was unilateral and only affected eyelids with skin-muscle resection (Table 1). The average amount of muscle removed in these patients was $15.3 \mathrm{~mm}$ and the mean age was 73.1 years (Table 1). In contrast, patients who did not demonstrate sluggish lid closure had a mean age of 56.8 years and had undergone an average of $8.3 \mathrm{~mm}$ skin-only or skinmuscle excision (Table 1). The increased incidence of sluggish

\section{Fig. 7. Preoperative photograph}

Before photograph of a 71-year-old patient undergoing bilateral upper blepharoplasties. Skin-only excision was randomly assigned to one eyelid and muscle-sparing excision randomly assigned to the other lid.

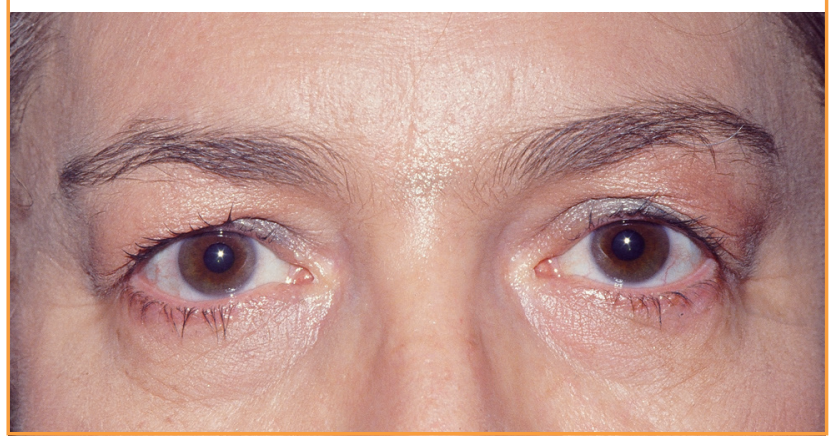

Fig. 8. Postoperative left-right comparison

Postoperative photograph of the patient in Fig. 7 six months after $13 \mathrm{~mm}$ of skin-only excision of the left lid and combined skin-muscle excision of the right upper eyelid. Patient also underwent concurrent lower blepharoplasties. This patient had temporary lagophthalmos and symptoms of dry eye on the right side that lasted less than four weeks. The panel of experts found "good but not comparable" outcomes on both sides and felt she could benefit from right eyebrow lift. The panel graded the outcome as "aesthetically good" on both sides.

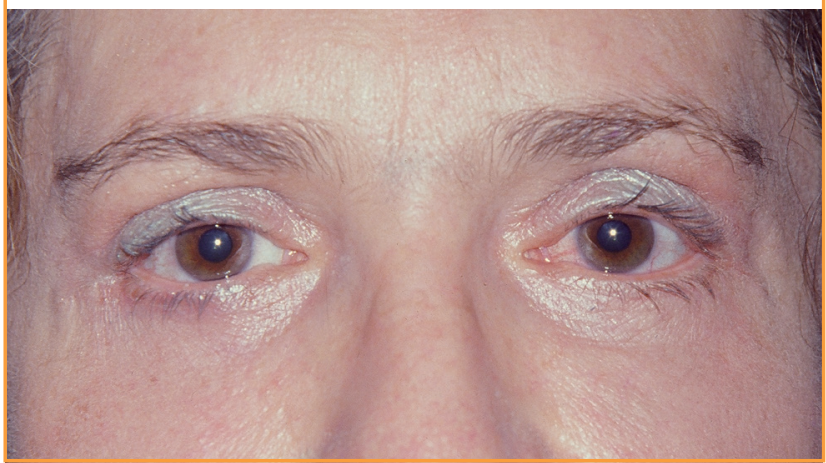


eyelid closure with skin-muscle removal compared to skin-only removal was statistically significant $(\mathrm{P}<0.05)$. The sluggishness persisted in all patients during the period of follow-up and was not found to be more severe in patients who had undergone tarsal fixation. This group of seven patients with sluggish eyelid closure included four of the five patients $(80 \%)$ who exhibited lagophthalmos of the ipsilateral eye postoperatively, and 5 of the $7(72 \%)$ patients who reported dry eye symptoms after surgery. None of the patients reported an awareness of their sluggish eyelid closure either during office visits or in their responses to

\section{Fig. 9. A case of pseudoptosis}

Before photograph of a 78-year-old patient with bilateral upper eyelid pseudoptosis due to excess skin. No true ptosis was present. Note patient's attempt to improve his visual field by elevating the eyebrows.

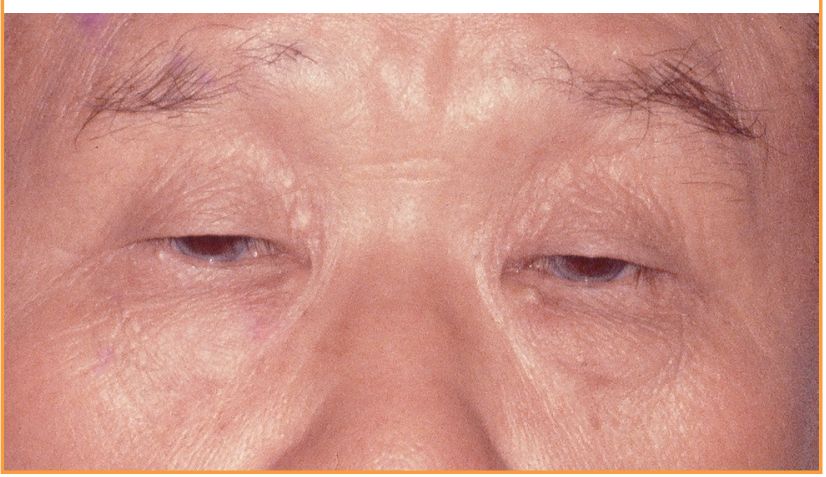

the mailed questionnaires six months after the procedures.

Lagophthalmos measuring less than $3 \mathrm{~mm}$ affected five patients ( $23 \%$ of the total patients) who had undergone an average of $15.8 \mathrm{~mm}$ of combined skin-muscle excision. It resolved in all patients within two to six weeks after surgery. All patients with lagophthalmos displayed a normal Bell's phenomenon on examination. Fig. 4 illustrates a 72-year-old patient seven days after

\section{Fig. 10. Postoperative left-right comparison}

Postoperative photograph of the patient in Fig. 9 six months after $21 \mathrm{~mm}$ of skin-only excision of the right lid and combined skinmuscle excision of the left upper eyelid. No tarsal fixation was performed. This patient had lagophthalmos of the eye that lasted one week but presented no dry eye symptoms. Note the absence of frontalis hyperactivity and dropping of the eyebrows. The panel of experts found the outcomes as "good and comparable" on both sides and graded the case as "aesthetically good".

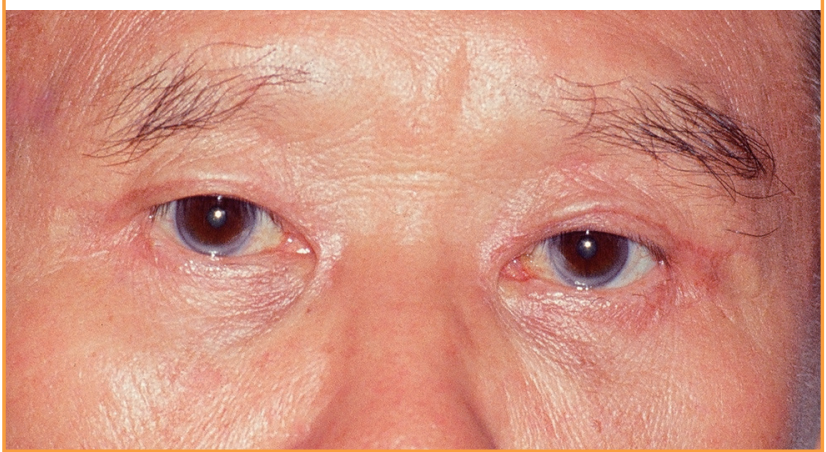

Table 1. Patient characteristics and postoperative findings and symptoms

\begin{tabular}{|c|c|c|c|c|c|c|c|c|}
\hline $\begin{array}{l}\text { Age } \\
\text { (yr) }\end{array}$ & $\begin{array}{c}\text { Width of } \\
\text { muscle } \\
\text { excision }(\mathrm{mm})\end{array}$ & $\begin{array}{l}\text { With tarsal } \\
\text { fixation }\end{array}$ & $\begin{array}{l}\text { Sluggish closure } \\
\text { skin-only } \\
\text { excision side }\end{array}$ & $\begin{array}{l}\text { Sluggish } \\
\text { closure skin- } \\
\text { muscle side }\end{array}$ & $\begin{array}{l}\text { Lagophthalmos } \\
\text { skin-only } \\
\text { excision side }\end{array}$ & $\begin{array}{l}\text { Lagophthalmos } \\
\text { skin-muscle } \\
\text { excision side }\end{array}$ & $\begin{array}{l}\text { Dry eye skin- } \\
\text { only excision } \\
\text { side }\end{array}$ & $\begin{array}{l}\text { Dry eye skin- } \\
\text { muscle } \\
\text { excision side }\end{array}$ \\
\hline 78 & 21 & & & $x$ & & $x$ & & \\
\hline 75 & 17 & & & $X$ & $X$ & $x$ & & $X$ \\
\hline 72 & 16 & & & $X$ & & & & \\
\hline 71 & 15 & $X$ & & $x$ & & $x$ & & $x$ \\
\hline 76 & 14 & & & $x$ & & & & $x$ \\
\hline 72 & 13 & & & $X$ & & $X$ & & $X$ \\
\hline 71 & 13 & & & & & $x$ & & $x$ \\
\hline 64 & 13 & & & & & & & \\
\hline 68 & 11 & $X$ & & $x$ & & & & $x$ \\
\hline 66 & 11 & & & & & & & \\
\hline 48 & 9 & $X$ & & & & & & $x$ \\
\hline 49 & 9 & & & & & & & \\
\hline 58 & 8 & & & & & & & \\
\hline 58 & 8 & $X$ & & & & & & \\
\hline 69 & 8 & $X$ & & & & & & \\
\hline 49 & 7 & $X$ & & & & & & \\
\hline 63 & 7 & $X$ & & & & & & \\
\hline 54 & 7 & $x$ & & & & & & \\
\hline 56 & 7 & $x$ & & & & & & \\
\hline 58 & 6 & & & & & & & \\
\hline 44 & 6 & $x$ & & & & & & \\
\hline 45 & 6 & & & & & & & \\
\hline
\end{tabular}


$13 \mathrm{~mm}$ of combined skin-muscle was removed from the right eyelid. She is unable to close the right eye despite her forceful attempt. In one 75 -year-old patient lagophthalmos was bilateral after $17 \mathrm{~mm}$ of excision, but more severe in the skin-muscle resection side than the skin-excision side (Table 1). In this patient, lagophthalmos lasted six weeks after surgery on the combined skin-muscle excision side but subsided within two weeks on the skin-only removal side. Concurrent lower blepharoplasty in several patients was not found to affect outcomes and no lower lid complication was documented. The higher incidence of lagophthalmos with skin-muscle excision compared to skin-only excision was statistically significant $(\mathrm{P}<0.05)$.

Varying degrees of dry eye symptoms such as foreign body sensation, tearing and irritation were reported in seven patients (32\%), as shown in Table 1. All these patients were followed for a minimum of six months. Five of these patients presented either lagophthalmos or sluggish lid closure or both (Table 1). In one patient, mild symptoms of eye irritation and rare itchiness were still present at one year after surgery; this patient was followed beyond the timeframe of this study. No dry eye symptoms were noted in eyes that underwent skin-only removal ( $\mathrm{Ta}$ ble 1). All patients with dry eye symptoms were treated with moisturizers and lubricants while the symptoms persisted, and all responded readily to standard care. Discontinuation of eye

\section{Table 2. Aesthetic outcome grades and scores}

\begin{tabular}{|c|c|c|c|}
\hline Outcome & Excision & $\begin{array}{l}\text { No. of } \\
\text { eyelids }\end{array}$ & $\begin{array}{l}\text { Average } \\
\text { score }^{\text {a) }}\end{array}$ \\
\hline \multirow[t]{2}{*}{ Aesthetically good } & Skin-only & 19 & 2.81 \\
\hline & Skin-muscle & 18 & 2.78 \\
\hline \multirow[t]{2}{*}{ Aesthetically acceptable to good } & Skin-only & 3 & 1.66 \\
\hline & Skin-muscle & 4 & 1.75 \\
\hline \multirow{2}{*}{ Aesthetically poor to acceptable } & Skin-only & 0 & 0 \\
\hline & Skin-muscle & 0 & 0 \\
\hline
\end{tabular}

a) "Aesthetically poor outcome" was graded as 1; "aesthetically acceptable outcome" was graded as 2; and "aesthetically good outcome" was graded as 3. The obtained grades were added, averaged and the final scores were interpreted as "aesthetically poor to acceptable outcome" when the average score was 1.00 to 1.50; as "aesthetically acceptable to good outcome" for the average scores of 1.51 to 2.00; as "aesthetically good outcome" when the average score was above 2.01 . care in two patients resulted in recurrence of symptoms and resolved with resumption of treatment.

Anonymous questionnaires were completed by 20 (91\%) patients. Of these 20 patients, 19 respondents were female and one was a male. The remaining two patients (9\%) did not respond to the questionnaire at six months after surgery and were lost to follow-up. The records, however, indicate that these patients were satisfied with the outcome and presented no abnormal findings and no symptoms at three months after surgery. Of the 19 female respondents, 15 women (79\%) expressed satisfaction from the procedures and found both sides comparable. In addition, four women (21\%) felt more skin should be removed from one of the eyelids. Of these four women, two expressed concern about the eyelid where only skin had been excised, while the other two women expressed concern about the eyelid where skin and muscle had been removed. In one female patient, simple excision of $2 \mathrm{~mm}$ of excess skin corrected the condition and satisfied the patient; during revision no muscle could be identified due to scar tissue present. In the other three women, although further excision was offered, they declined revision and in time felt that the results are good and acceptable. Although seven patients complained of dry eye symptoms during their office visits, only one patient made mention of dry eyes in the questionnaire.

Patients' photographs (Figs. 5-10) were reviewed by a panel of three plastic surgeons. For two patients, the reviewed photographs were taken at three months after surgery since they were lost to follow-up. However, in the remaining 20 (91\%) patients, photographs taken at six months after surgery were available. The panelists found fellow eyelids to be comparable in 18 of 22 cases $(82 \%)$ and rated the results as "good and comparable". In four patients, more than one panelist recommended that more skin could have been removed (two on the skin-only side and two on the skin-muscle side to accomplish better symmetry). For five patients, two panelists felt that eyebrow lift (an exclusion criteria in this study) could be beneficial if they choose to proceed with such an operation in the future (Figs. 6,8 ). No statistical difference in aesthetic outcome was found between eyelids that had undergone skin excision alone and eyelids with

Table 3. Postoperative complications after combined skin-muscle excision

\begin{tabular}{|c|c|c|c|c|c|c|c|c|c|c|c|c|c|c|c|c|c|c|c|c|c|c|}
\hline \multirow{2}{*}{ Complications } & \multicolumn{22}{|c|}{ Width of muscle excision (mm) } \\
\hline & 6 & 6 & 6 & 7 & 7 & 7 & 7 & 8 & 8 & 8 & 9 & 9 & 11 & 11 & 13 & 13 & 13 & 14 & 15 & 16 & 17 & 21 \\
\hline Sluggishness & & & & & & & & & & & & & & $x$ & & & $x$ & $x$ & $x$ & $x$ & $x$ & $x$ \\
\hline Lagophthalmos & & & & & & & & & & & & & & & & $x$ & $x$ & & $x$ & & $X$ & $x$ \\
\hline Dry eye & & & & & & & & & & & & $x$ & & $x$ & & $x$ & $x$ & $x$ & $x$ & & $x$ & \\
\hline
\end{tabular}

This table shows the relationship between the width of muscle excision in $\mathrm{mm}$ and subsequent complications of sluggishness of lid closure, lagophthalmos and dry eye symptoms following upper blepharoplasties. 
combined skin and muscle removal (Table 2).

\section{DISCUSSION}

Despite the senior author's very busy aesthetic blepharoplasty practice, only 22 patients consented. This limited prospective study strongly suggests there is no advantage in excising the orbicularis muscle as part of "standard" upper blepharoplasty. By survey and physical exam, we identified long-term complications of surgery with muscle resection exceeding $9 \mathrm{~mm}$, namely sluggish lid closure, lagophthalmos and varying degrees of dry eye symptoms (Tables 1, 3); we consider the introduction of "Sluggish lid closure" as original and of great clinical significance not only in our clinical practice but also in future studies. Since the aesthetic outcomes of skin-only resection were equivalent to those eyelids that had undergone combined skin-muscle excision as demonstrated both by patient satisfaction surveys and by examination of the postoperative photographs by a panel of experts (Table 2), we recommend that muscle resection is best avoided. The limited cohort of patients included both Asian eyelids without a defined supratarsal fold as well as eyelids with well-defined supratarsal crease. Eyelids underwent tarsal fixation from both these groups of patients and yet no difference in aesthetic or functional outcomes was noted.

In 2011, Damasceno et al. [12] reported that postoperative edema, hematoma, itching and pain were greater with skin-muscle resection. These complications were transient with resolution at 30 days. Similar to our study, the authors also found that eventual aesthetic outcomes were equivalent in skin-only and skin-muscle resections [12]. The duration of dry eye symptoms following skin-muscle resection was longer in our study and lasted throughout the follow-up period of six months in patients who had undergone wider than $9 \mathrm{~mm}$ of muscle excision. Damasceno et al. failed to document the amount of muscle removed and made no mention of effects on lid function, or aesthetic importance that correlates with the extent of muscle excision. Damasceno et al. studied 15 consecutive patients over a short period of time and no attempt was made to select patients who may require significant muscle excision. We differ importantly from Damasceno et al. in the fact that in our study 12 out of 22 volunteers underwent wider than $9 \mathrm{~mm}$ of muscle excision. Our patient selection represented a painstaking task requiring 27 years to complete due to difficulty in obtaining volunteers for this left-right prospective study. Our finding that warns clinicians to avoid excisions of muscle and definitely not more than 9 millimeters wide when performing upper blepharoplasty is significant and not reported previously.

Our study also indicates that older patients are at increased risk for sluggish eyelid closure as well as lagophthalmos, and that these complications are related to the amount of orbicularis muscle excised. Furthermore, sluggish lid closure and lagophthalmos are closely related since $57 \%$ of patients with sluggish eyelid closure also had lagophthalmos, and $80 \%$ of patients with lagophthalmos presented with sluggish eyelid closure (Table 1). Sluggish lid closure and lagophthalmos are significant in that they may increase the risk of corneal injury. In order to followup and assess the risks of such injury, we emphasize the importance of examining the patients after surgery not only with eyes open, but also with eyes gently closed, in extreme downward gaze and simulating a sleep state. One must note any sluggish lid closure as well as presence or absence of Bell's phenomenon when lagophthalmos is observed.

Although lagophthalmos gradually subsided during the period of this study, sluggish lid closure seemed unchanged during the same period and appears to play a role as significant as lagophthalmos in causing dry eye symptoms. It is possible that the temporary effect of the surgical trauma and postoperative edema play a significant role in the duration of lagophthalmos, while it is the absence of muscular fibers that play a critical role in sluggish lid closure that shows no observable improvement during the same period of observation.

Our expert panel identified five patients who could benefit from a future brow-lift procedure following their upper blepharoplasty procedure (Figs. 6, 8). In the setting of lid ptosis or pseudoptosis (Fig. 9), it is known that the brows may be raised by frontalis action in order to increase the visual fields. The correction of pseudoptosis allows the brows to fall as shown in Figs. 9,10 . Therefore, it is advisable that patients be made aware of the possible need for subsequent brow-lift when being consented for aesthetic upper blepharoplasty.

The number of cohorts constitutes the main limitation of this prospective report. Lack of video documentation is another weakness in our study. Videography and electromyography would be superior means to document dynamic differences in eyelid closure than mere observation, as utilized in this study. Evaluation of dry eye symptoms could be better measured using methods such as tear osmolarity, tear lysozyme and fluorescein disappearance testing. Another weakness is that a portion of the study was not double-blinded: the primary surgeon (F.D.P.) was aware of the side with muscle resection, as he followed the patients postoperatively.

Muscle-sparing upper blepharoplasty has equivalent aesthetic outcomes to conventional surgery with muscle resection, and decreases the risk for corneal surface exposure and its sequelae. We found that muscle resection does not improve the aesthetic outcome, but may increase the morbidity. To recognize and ini- 
tiate early treatment of dry eye disease following upper blepharoplasty, the surgeon should be vigilant in observing eyelids while open, gently closed, and in motion so that proper intervention to maintain the tear film may be implemented.

\section{REFERENCES}

1. Rohrich RJ, Coberly DM, Fagien S, et al. Current concepts in aesthetic upper blepharoplasty. Plast Reconstr Surg 2004; 113:32e-42e.

2. Fagien S. Advanced rejuvenative upper blepharoplasty: enhancing aesthetics of the upper periorbita. Plast Reconstr Surg 2002;110:278-91.

3. Hoorntje LE, Lei B, Stollenwerck GA, et al. Resecting orbicularis oculi muscle in upper eyelid blepharoplasty: a review of the literature. J Plast Reconstr Aesthet Surg 2010;63:78792.

4. Zide BM, Jelks GW. Surgical anatomy of the orbit. New York: Raven Press; 1985.

5. Terella AM, Wang TD, Kim MM. Complications in periorbital surgery. Facial Plast Surg 2013;29:64-70.

6. Sheen JH. Supratarsal fixation in upper blepharoplasty. Plast Reconstr Surg 1974;54:424-31.
7. Januszkiewicz JS, Nahai F. Transconjunctival upper blepharoplasty. Plast Reconstr Surg 1999;103:1015-8.

8. Har-Shai Y, Hirshowitz B. Extended upper blepharoplasty for lateral hooding of the upper eyelid using a scalpel-shaped excision: a 13-year experience. Plast Reconstr Surg 2004; 113:1028-35.

9. Lelli GJ Jr, Lisman RD. Blepharoplasty complications. Plast Reconstr Surg 2010;125:1007-17.

10. Carroll RP, Mahanti RL. En bloc resection in upper eyelid blepharoplasty. Ophthal Plast Reconstr Surg 1992;8:47-9.

11. Thomas CB, Perez-Guisado J. A new approach: resection and suture of orbicularis oculi muscle to define the upper eyelid fold and correct asymmetries. Aesthetic Plast Surg 2013;37:46-50.

12. Damasceno RW, Cariello AJ, Cardoso EB, et al. Upper blepharoplasty with or without resection of the orbicularis oculi muscle: a randomized double-blind left-right study. Ophthal Plast Reconstr Surg 2011;27:195-7.

13. Parsa AA, Lye KD, Radcliffe N, et al. Lower blepharoplasty with capsulopalpebral fascia hernia repair for palpebral bags: a long-term prospective study. Plast Reconstr Surg 2008; 121:1387-97. 